\title{
On the Performance of Digital Back Propagation in Spatial Multiplexing Systems
}

\author{
Filipe M. Ferreira, Senior Member, IEEE, Stylianos Sygletos, Eric Sillekens, Student Member, IEEE, \\ Robert Killey, Senior Member, IEEE, Andrew D. Ellis, Nick J. Doran
}

\begin{abstract}
Nonlinear performance in spatial multiplexing systems is strongly determined by the interplay between differential mode delay, linear mode coupling and Kerr nonlinearity. In this work we review and extend the analysis of different solution methods for the linear coupling operator in the coupled nonlinear Schrödinger equation for spatial multiplexed propagation. Numerical solution methods are compared for different operational regimes as determined by differential mode delay and linear mode coupling. Finally, we review and extend the study of digital methods to mitigate the Kerr nonlinearity for arbitrary levels of random linear mode coupling. For the first time, it is shown that in spatial multiplexing systems transmission performance can be improved by reducing the number of back propagated channels for non-negligible levels of differential mode delay.
\end{abstract}

Index Terms-Digital-Back Propagation, Linear Mode Coupling, Spatial Division Multiplexing.

\section{INTRODUCTION}

$\mathrm{S}$ Patial-Division MultiPleXING (SDM) has emerged as one possible solution to overcome the capacity limit of single-mode fibers (SMFs) [1]. Among the SDM approaches offering the highest spatial information density there are two prime candidates: few-mode fibers (FMFs) and coupled-core multi-core (CC-MCFs). They offer a number of advantages, such as lower nonlinear coefficients; higher pump efficiency for optical amplification (similar to core pumped SMF) [2]; higher spatial-density integration in transponders [3], amplifiers, and add-drop multiplexers (multiple spatial modes can be routed together [4]). However, the multitude of spatial modes introduces new impairments, namely: group delay (GD) spread [5-10] given the interplay between differential mode delay (DMD) and linear mode coupling (LMC), inter-modal nonlinear effects (IM-NL) [11-16], and mode dependent loss (MDL) [17-19]. Chief among these is the LMC that plays a crucial role at controlling the GD spread, MDL accumulation and the efficiency of the overall nonlinear interactions. Thereby, and with practical fibers

Manuscript received day month, year; revised day month, year; accepted day month, year. Date of publication day month, year; date of current version day month, year. This work has been funded by the Engineering and Physical Sciences Research Council (Grants EP/R024057/1-FPA-ROCS, EP/M005283/1-UPON, EP/S003436/1-PHOS， EP/T009047/1-PON-HD, EP/R035342/1-TRANSNET).

F. Ferreira, Eric Sillekens and Robert Killey are with the Optical Networks Group, Department of Electronic \& Electrical Engineering, University College operating in all LMC regimes [20-26], the modelling of LMC has been under intensive research [5-10] as well as its impact on the statistics of GD, MDL and NL.

For systems operating in the linear regime the GD spread can be fully overcome using multi-input multi-output (MIMO) based DSP techniques [27, 28], and DMD compensation maps for long-haul transmission over 1000s km [29, 30]. Currently, performance is mainly limited by prototype components MDL [31] and by fiber IM-NL interactions [32, 33]. But given the continuous improvement of mode/core multiplexers [34-38] the impact of IM-NL will become dominant. Here we demonstrate the applicability of digital back propagation (DBP) to address the IM-NL penalties in SDM systems. To allow a direct performance assessment of the nonlinear performance, MDL is not considered in this paper. MDL is studied in detail in [17-19].

This paper is organized as follows. Section II presents some of the most representative models for nonlinear transmission over SDM fibers in all LMC regimes. Section III presents the wavelength division multiplexing (WDM) SDM system to be used in this paper. Section IV presents and analyzes simulation results for the different transmission models in section II over a wide range of $D M D$ and $X T$ values; extending our previous results by considering a larger number of WDM channels over span lengths optimized to minimize power consumption [39, 40]. Section V extends our recent results on the application of DBP to WDM-SDM systems [41-43] by: (i) analyzing the dependency on the number of back-propagated channels; (ii) considering a wider range of LMC and DMD scenarios; (iii) extending the characterization of the GD spreading in the strong LMC regime. Section VI draws the final conclusions.

\section{LiNEAR MODE COUPLING AND NONLINEAR TRANSMISSION MODELLING}

\section{A. Linear Mode Coupling Regimes}

Three LMC regimes are usually identified: weak coupling (WC), strong coupling (SC) and intermediate coupling [9]. WC usually refers to the case of 2 (or more) non-degenerate mode groups whose inter-group coupling can be neglected over the

London, London WC1E 7JE, UK (e-mail: f.ferreira@ucl.ac.uk; e.sillekens@ucl.ac.uk; rkilley@ee.ucl.ac.uk). F. Ferreira was with the Aston Institute of Photonic Technologies, School of Engineering and Applied Science, Birmingham B4 7ET, UK.

S. Sygletos, A. Ellis and N. Doran are authors are with the Aston Institute of Photonic Technologies, School of Engineering and Applied Science, Birmingham B4 7ET, UK (e-mail: s.sygletos@aston.ac.uk; andrew.ellis@aston.ac.uk; n.j.doran@aston.ac.uk). 
distance of interest; likely to be the case in (multiple-)stepindex [24, 44] or sparsely-spaced MCFs [45]. Intermediate coupling generally applies to FMFs supporting several nondegenerate mode groups whose inter-group coupling cannot be neglected for the transmission distance of interest; likely to be the case for graded-core fibers optimized for low $D M D[21,25]$. SC usually applies to the coupling between (quasi-)degenerate modes within a given mode group of a FMF, or the coupling between in CC-MCFs [26], or simply the coupling between the two polarization modes in SMFs.

Quantitatively, the LMC regime in which a fiber link is operating is determined by the coupling length or the coupling strength; figures of merit originally proposed for polarization mode dispersion in SMFs [46-48] that can be extended for SDM fibers. The coupling strength $(X T)$ is quantified as the ratio between the sum of the average power $(P)$ in all the other orthogonal modes and the average power remaining in the launching mode $(m)$ after a certain distance $(z)$, $X T(z)=\sum_{v \neq m}\left[P_{v}(z) / P_{m}(z)\right]$. And, the coupling length $\left(L_{c}\right)$ is quantified as the length for which the average power in the orthogonal modes is within $e^{-2}$ of the power in the launching mode, this is equivalent to $X T\left(L_{c}\right)=\left[e^{2}-1\right] /\left[e^{2}+1\right](-1.18 \mathrm{~dB})$. Fig. 1 shows the accumulated $X T$ as a function of the transmission distances in an arbitrary fiber for three different launch modes each with a different $X T$ per unit of length ([-30, $-40,-50] \mathrm{dB} / \mathrm{m})$. It is clear from the figure that in the case of multi-mode (or -core) there are as many $L_{c}$ values as modes (or cores). Therefore, the launching mode $m$ presenting higher coupling strength is used as the refence to calculate $X T$ or $L_{c}$; $\mathrm{LP}_{02}$ for the fiber used in this paper. Finally, after a certain transmission distance $(L)$, a fiber link is said to be operating in the: SC-regime for $L \gg L_{c}$, WC-regime for $L \ll L_{c}$ and intermediate regime for $L \sim L_{c}$.

\section{B. GD Spread}

In SDM fibers, the temporal spread of a propagating pulse is determined by the GD vector $\tau$, as defined for a generalized $\left(M^{2}-1\right)$-dimensional Stokes space in [49], where $M$ is the number of modes. Knowledge of the modulus of the GD vector $\|\boldsymbol{\tau}\|$ allows to determine the GD spread [49]. For non-DMDmanaged spans the GD spread $(T)$ after a certain transmission distance $(z)$ is given by:

$$
T^{2}(\mathrm{z})=\left\langle\|\boldsymbol{\tau}(z)\|^{2}\right\rangle /(2 M)^{2}=2\left\|\partial_{\omega} \boldsymbol{\beta}\right\|^{2} L_{c}{ }^{2}\left(e^{-z / L_{c}}+z / L_{c}-1\right)
$$

where the operators $\langle$.$\rangle and \|$.$\| denote expectation and$ modulus, respectively; $\partial_{\omega} \boldsymbol{\beta}$ term represents the uncoupled GDs per unit length assumed constant along $z$, considering the same $L_{c}$ for all groups of modes. Equation (1) was proposed and validated by simulation in [50] and concurrently analytically derived in [8].

In this paper, for links operating in the WC- or intermediate regime, we will describe the temporal spread of a propagating pulse by referring to $D M D$ - the delay difference between the maximum and minimum of the uncoupled $G D$ vector. However, for links in the SC-regime, we will describe temporal spread by referring to the GD spread in (1).

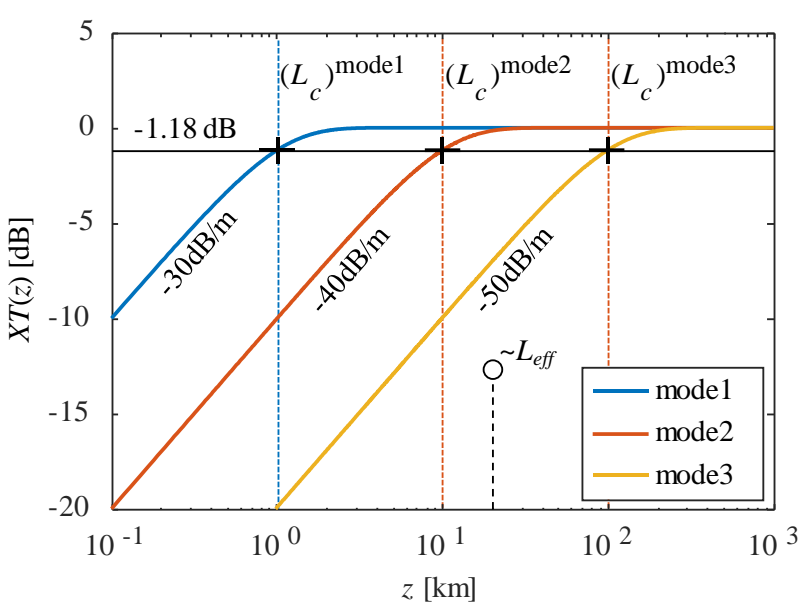

Fig. 1. Accumulated $X T$ as a function of the fiber length (z) for different launch modes, each with a different $L_{c}$, in an arbitrary fiber.

\section{Nonlinear Transmission Modelling}

Modelling nonlinear SDM transmission including LMC involves solving a coupled nonlinear Schrödinger equation (CNLSE), which can be written as [11-16]:

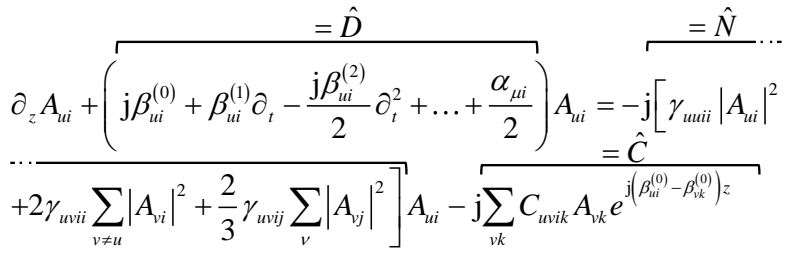

for the slowly varying field envelope component $A_{u i}(z, t)$ in the mode $u$ and the polarization $i$. The terms $\beta_{u i}{ }^{(p)}$ and $\alpha_{u i}$ indicate the $p^{\text {th }}$-derivative with respect to the angular frequency of the mode propagation constant and the attenuation, respectively, of the mode $u$ and polarization $i$. The terms $C_{u v i j}$ and $\gamma_{u v i j}$ indicate the nonlinear and the LMC, respectively, between the $i$-th polarization of the mode $u$ and the $j$-th polarization of the mode $v$. Note that the non-italic letter " $\mathrm{j}$ " is reserved for the imaginary unit $(-1)^{1 / 2}$. Equation (2) can be rewritten in terms of the operators: dispersion $\widehat{D}$, nonlinearity $\widehat{N}$ and LMC $\hat{C}$.

The numerical integration of (2) can be achieved considering these three operators, dispersion, LMC and nonlinearity, acting independently for a sufficiently short integration step. The step must be much shorter than: (i) the dispersion length $T_{0}{ }^{2} / \beta_{u}{ }^{(2)} \mid$; (ii) the walk-off length $T_{0} / \beta_{u}{ }^{(1)}-\beta_{v}{ }^{(1)} \mid\left(T_{0}\right.$ is the bandwidth reciprocal); (iii) the correlation length $L_{c}$; (iv) the nonlinear effective length $L_{\text {eff }}=[1-\exp (-\alpha L)] / \alpha, \alpha$ is the attenuation coefficient and $L$ is the span length. For the numerical integration of (2), the LMC operator can be resolved in two ways: numerically, having to generate random coupling matrices every step with a given coupling strength; analytically via Manakov equations derived by averaging the nonlinear operator over all possible LMC realizations. In the numerical approach there are two main variants referred here as: lumped $L M C$ and distributed LMC. In the following, numerical and analytical handlings of LMC in nonlinear transmission are reviewed.

In all cases, to solve (2) we use a symmetric implementation of the split-step Fourier method [51], the nonlinear step is in the middle of the segment rather than at the segment boundary. The 
step-size is adapted by bounding the local error to be smaller than $10^{-5}$ (smaller values led to negligible change).

\section{Lumped LMC Modelling}

In this case, multi-section modelling includes LMC among nondegenerate modes via random unitary matrices with a section length just longer than $L_{c}$ such that accumulated $X T$ equals $0 \mathrm{~dB}$ in average $[6,10]$, see Fig. 1. This approach is well suited for the linear power regime allowing matching the analytical predictions for GD statistics [6-8], provided that $L_{c}$ is much shorter than the dispersion length and the walk-off length. In the nonlinear power regime, this model is restricted to SDM fibers that operate in the intermediate-to-strong LMC regime. This is, for fibers achieving full mode mixing for distances smaller than the $L_{\text {eff }}(20 \mathrm{~km}$ in typical cases), see Fig. 1 . Nevertheless, this model is still applicable to SDM fibers in the WC-regime for which LMC between non-degenerate can be neglected.

\section{E. Distributed LMC Modelling}

A semi-analytical model capable of describing the LMC for fibers operating in the intermediate coupling regime has been developed by the authors in [5, 9]. In this model all LMC is assumed to arise from core-cladding imperfections which are discretized by dividing the fiber in multiple sections, each with a random displacement of the core-center position. This simplification allows deriving semi-analytical solutions of the LMC operator that can be used in the numerical integration of the CNLSE (2). The LMC strength is set using a fixed amount of radial displacement and a random azimuth displacement given by a uniform distribution. In this way, it was shown in [9] that at each step a random amount of LMC is introduced among non-degenerate modes that in average approximates the desired level. This method has been proven accurate in the linear power regime, matching the analytical GD statistics in FMF links for transmission lengths up to $10,000 \mathrm{~km}$, in any LMC regime, with and without GD management [9]. In section IV, the applicability of this model to nonlinear propagation in SDM fibers is tested.

\section{F. Manakov Equations}

Analytically, and in the presence of extreme LMC regimes, it has been shown [11-13] that some or all the LMC terms in the CNLSE (2) can be assumed to vary rapidly and seemingly randomly on a length scale that is expected to be short compared to the effective lengths associated with chromatic dispersion and the various manifestations of nonlinearity. Thus, like in SMFs and the well-known Manakov-PMD equations [52], one can average the propagation equation (2) over all possible realizations of the LMC operator $\hat{C}$. This way, the LMC operator $\hat{C}$ disappears from (2) and the nonlinear operator $\widehat{N}$ is replaced by an averaged equivalent. The nonlinear operator was averaged for the two extreme coupling regimes in SDM fibers.

In the weak coupling (WC) regime [11], inter-mode-group coupling is neglected and intra-mode-group coupling is assumed strong. Averaging over all possible realizations of

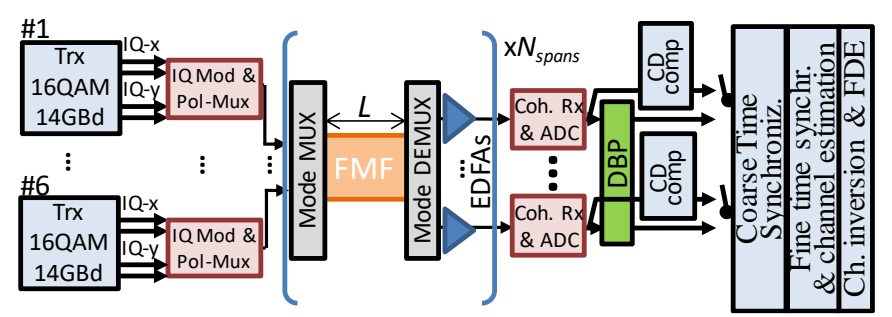

Fig. 2. Block diagram for system simulations using a fibre with $6 \mathrm{LP}$ modes each with 2 orthogonal polarizations.

TABLE I. FIBRE LINEAR CHARACTERISTICS AT 1550NM.

\begin{tabular}{|l|c|c|c|c|c|c|}
\hline$u$ & LP01 & LP02 & LP11a & LP11b & LP21a & LP21b \\
\hline$\beta_{u}{ }^{(1)}[\mathrm{ps} / \mathrm{km}]$ & -0.29 & -2.93 & -0.66 & -0.66 & 2.27 & 2.27 \\
\hline$B_{u}{ }^{(2)}\left[\mathrm{ps}^{2} / \mathrm{km}\right]$ & -28.28 & -27.48 & -28.25 & -28.25 & -27.86 & -27.86 \\
\hline$\beta_{u}{ }^{(3)}\left[\mathrm{ps}^{3} / \mathrm{km}\right]$ & 0.15 & 0.15 & 0.15 & 0.15 & 0.15 & 0.15 \\
\hline$\alpha_{u}[\mathrm{~dB} / \mathrm{km}]$ & 0.2 & 0.2 & 0.2 & 0.2 & 0.2 & 0.2 \\
\hline
\end{tabular}

TABLE II. NONLINEAR COEFFICIENTS* $\left(\gamma_{w v}\right)\left[\mathrm{W}^{-1} / \mathrm{KM}\right]$ AT $1550 \mathrm{NM}$.

\begin{tabular}{|l|c|c|c|c|c|c|}
\hline$u$ & LP01 & LP02 & LP11a & LP11b & LP21a & LP21b \\
\hline LP01 & 0.73 & 0.36 & 0.36 & 0.36 & 0.18 & 0.18 \\
\hline LP02 & 0.36 & 0.36 & 0.18 & 0.18 & 0.18 & 0.18 \\
\hline LP11a & 0.36 & 0.18 & 0.55 & 0.18 & 0.27 & 0.27 \\
\hline LP11b & 0.36 & 0.18 & 0.18 & 0.55 & 0.27 & 0.27 \\
\hline LP21a & 0.18 & 0.18 & 0.27 & 0.27 & 0.41 & 0.14 \\
\hline LP21b & 0.18 & 0.18 & 0.27 & 0.27 & 0.14 & 0.41 \\
\hline
\end{tabular}

$*$ Note that $\gamma_{u v i j}=\gamma_{u v i i}=\gamma_{u w}$, the degeneracy factors are explicitly considered in (2), (3) and (4).

intra-mode-group random coupling, the intra-modal and intermodal degeneracy factors in (2) become:

$$
\begin{aligned}
& \hat{N}=-\mathrm{j} \sum_{\substack{v \\
k=\{i, j\}}} \kappa_{u v}\left|A_{v k}\right|^{2}, \\
& \kappa_{u u}=\frac{4}{3} \frac{2 M_{u}}{2 M_{u}+1} \bar{\gamma}_{u u}, \quad \kappa_{u v}=\frac{4}{3} \bar{\gamma}_{u v}(u \neq v), \\
& \bar{\gamma}_{u v}=\frac{1}{M_{u} M_{v}} \sum_{u, v} \gamma_{u v v u}
\end{aligned}
$$

In the strong coupling (SC) regime, it is considered that all modes are strongly linearly coupled. In this case, the nonlinear operator in (2) becomes [12]:

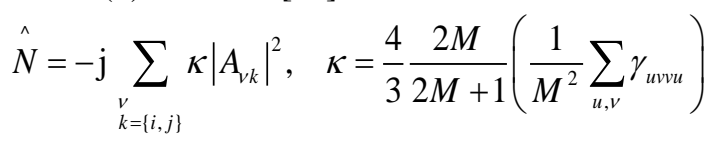

In deriving (3) and (4), it is assumed that spatial modes that are strongly coupled propagate with similar group delay (that is, $D M D$ should not be higher than a few $\mathrm{ps} / \mathrm{km}$ ). This is likely the case for degenerate modes in FMFs or coupled-core MCFs [26, 29]. However, extension of the Manakov models for the general case in terms of $L M C$ and $D M D$ is still under investigation [53].

In the SC-regime, the Manakov equation provides a significant advantage in terms of integration time w.r.t. the distributed LMC approach, since the latter requires step size with average $X T$ smaller than $-20 \mathrm{~dB}$, while the Manakov equation model does not. Conversely, in the other regimes, the two approaches are comparable in terms of integration time, as discussed in section IV of [54]. 


\section{Simulation Setup}

In this section we describe the WDM-SDM transmission setup proposed to evaluate different transmission models (section IV) and to evaluate the potential for DBP (section V).

The simulation setup is shown in Fig. 2. A mode-divisionmultiplexing system using a FMF with 6 linearly polarized (LP) modes $\left(\mathrm{LP}_{01}, \mathrm{LP}_{02}, \mathrm{LP}_{11 \mathrm{a}}, \mathrm{LP}_{11 \mathrm{~b}}, \mathrm{LP}_{21 \mathrm{a}}\right.$ and $\left.\mathrm{LP}_{21 \mathrm{~b}}\right)$ each with 2 orthogonal polarizations is considered. The fiber was optimized in [55] for low DMD; linear and nonlinear characteristics at $1550 \mathrm{~nm}$ are given in table I and II, respectively. Transmission simulations consider an optical super-channel with a varying number of WDM channels (per mode) modulated with 14 Gbaud polarization-multiplexed 16QAM, $14.1 \mathrm{GHz}$ spaced; the line rate per channel is $672 \mathrm{Gbit} / \mathrm{s}$.

Together with the information data, a preamble is transmitted consisting of constant amplitude zero autocorrelation (CAZAC) sequences, used for time synchronization and channel estimation. Root raised cosine filters with a roll-off factor of 0.001 is used for pulse shaping. Simulations considered $2^{16}$ symbols per polarization mode, the first $2^{12}$ were CAZAC symbols and the remaining were 16-QAM symbols mapped from a subset of a $2^{23}-1$ PRBS [56]. The fiber attenuation is fully compensated using an array of 6 erbium doped fiber amplifiers, considering a noise figure of $3 \mathrm{~dB}$ and negligible mode dependent gain since the aim of this section is to assess the impact of $L M C$ and $D M D$ on the accuracy of the different solution methods. Similarly, the mode multiplexer (MUX) and de-multiplexer (DEMUX) were assumed to be ideal for the same reasons. After homodyne detection, the baseband electrical signals are sampled at $2 \times N_{D B P}$ samples/symbol (where $N_{D B P}$ stands for the number of channels back propagated), yielding 12 digital signals (2 polarizations times 6 modes). Afterwards, the coherently received signals are either (i) compensated for chromatic dispersion in the frequency domain using the values in the Table I or (ii) DBP compensated for chromatic dispersion and nonlinear distortion $[57,58]$ using a virtual fibre with characteristics of opposite-sign values of those in the Table I and II, except that no mode coupling is considered and the uncoupled GD vector is neglected for $X T>-40 \mathrm{~dB} / \mathrm{m}$. In all cases, $L M C$ and (residual) $D M D$ were subsequently compensated using data-aided channel estimation and equalization, as shown in Fig. 2. Coarse time synchronization is performed using the Schmidl \& Cox autocorrelation metric. Subsequently, fine-time synchronization and channel impulse response (CIR) estimation are performed by cross-correlating with the training CAZAC sequences. The $12 \times 12$ CIR estimations are converted into the frequency domain. The MIMO frequency domain equalizer is calculated by inverting the estimated channel matrix, and, finally, the signal-to-noise-ratio of the channel of interest is estimated [59].

The figure of merit in the following is the minimum signalto-noise-ratio (SNR) among the 12 polarization modes guided of the center wavelength channel. The SNR is evaluated as [59] the ratio between the variance of the transmitted symbols $E\left[|X|^{2}\right]$ and the variance of the noise $E\left[|X-Y|^{2}\right], X$ and $Y$ represent the received symbols, respectively. However, in [59] Sec. V-B the authors explain that treating samples from a nonlinear channel (the case in this paper) as additive white Gaussian noise

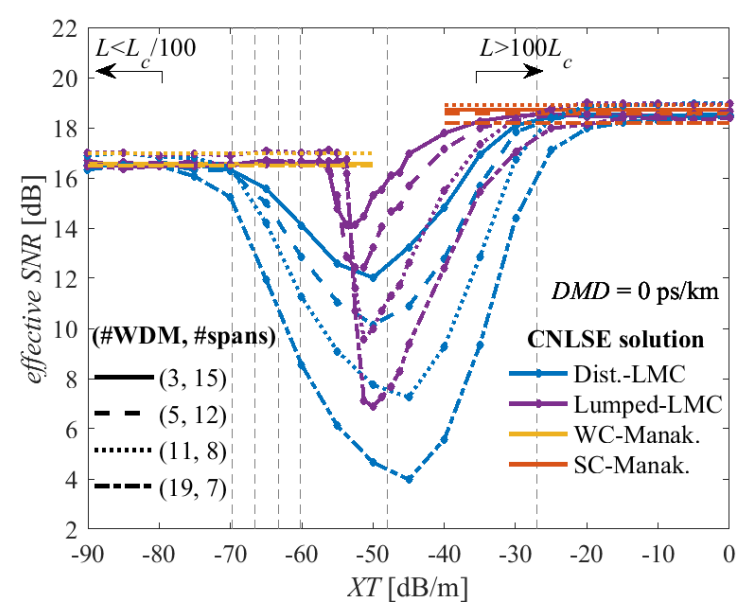

Fig. 3. Effective $S N R$ as a function of $X T$ at $0 \mathrm{dBm} / \mathrm{ch}$ with $D M D=0 \mathrm{ps} / \mathrm{km}$, for: 3 channels over 15 spans, 5 channels over 12 spans, 11 channels over 8 spans and 19 channels over 7 spans. Data points averaged over 10 repetitions. Each of the six dashed vertical lines identify one of the fibers studied in [20-25], respectively, from left to right.
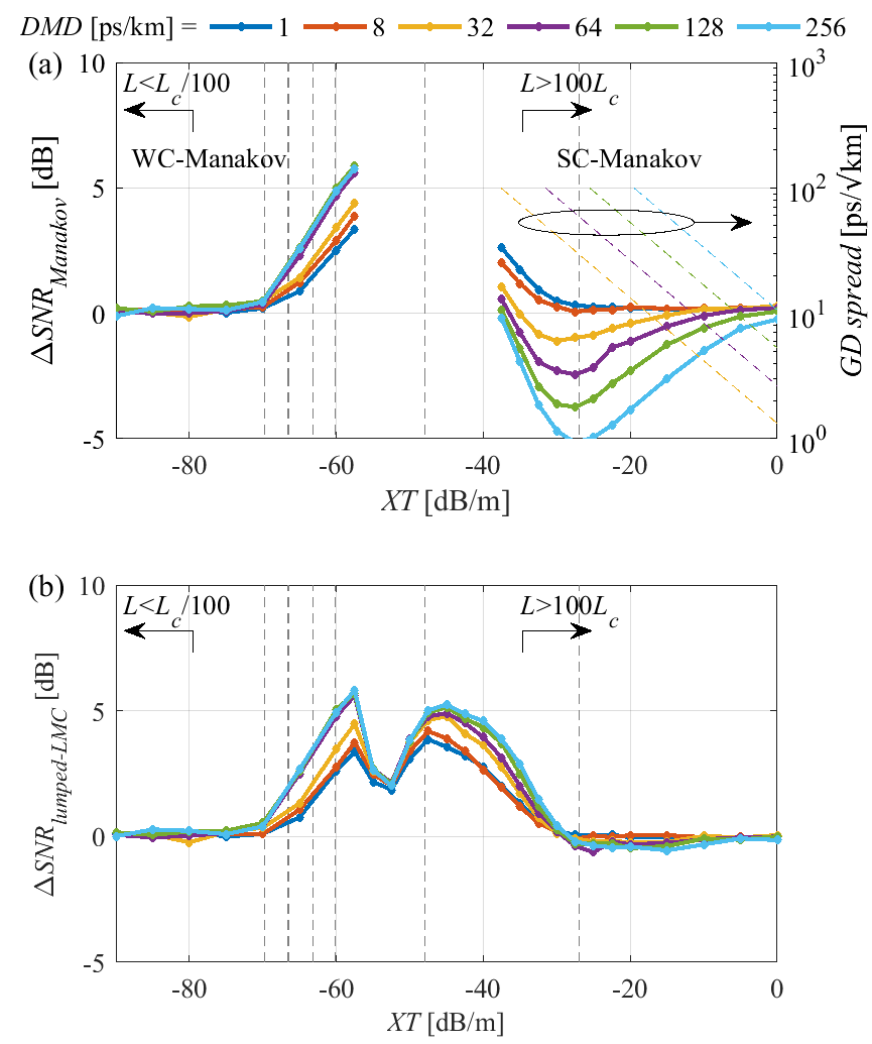

Fig. 4. Effective $S N R$ error as a function of $X T$ using the distributed LMC model as reference $(0 \mathrm{dBm} / \mathrm{ch}, 3$ channels and 15 spans) for different models: (a) WCand SC-Manakov, and (b) lumped LMC. Each of the six dashed vertical lines identify one of the fibers studied in [20-25], respectively, from left to right.

(AWGN) samples gives a lower bound on the performance. This is, system implementations are expected to perform the same or better compared to an AWGN channel with the same SNR. For this reason the term effective SNR is used in the following. Finally, system performance simulations consider spans of $35 \mathrm{~km}$, the optimum length to minimize the total energy requirement for amplified systems with a fibre loss of $0.2 \mathrm{~dB} / \mathrm{km}$. We followed the design rule derived in [60], this is: 
the optimum length is given by $2+W\left(-2 / \mathrm{e}^{2}\right) / \alpha$, where $W($.) represents the Lambert $\mathrm{W}$ function.

\section{TRANSMISSION MODELS COMPARISON}

This section compares the models discussed in section II for forward transmission simulation, considering the system in section III without DBP (see Fig. 2), namely: WC-Manakov, SC-Manakov, distributed LMC, and lumped LMC.

Fig. 3 shows effective SNR as a function of $X T$, in the nonlinear regime $0 \mathrm{dBm} /$ channel and absence of $D M D$, for different numbers of WDM channels and link spans, namely: 3 channels over 15 spans, 5 channels over 12 spans, 11 channels over 8 spans and 19 channels over 7 spans. Dashed vertical lines indicate the $D M D$ of the experimental fibers studied in [20-25], respectively from left-to-right. Manakov results are independent of $X T$, but to identify their domain of applicability horizontal arrows mark the $X T$ range over which the total transmission distance greater than $100 \cdot L_{c}$ or smaller than $L_{c} / 100$. Moreover, note that for an increasing number of channels, the number of spans was reduced such that the performance given by the WC-Manakov model remained similar; for the sole purpose of simplifying the visualization of the results. The results in Fig. 3 show an excellent agreement between the Manakov models and the lumped LMC and the distributed LMC models in the extreme LMC regimes. However, in the intermediate LMC regime $(-70 \mathrm{~dB} / \mathrm{m}$ to $-30 \mathrm{~dB} / \mathrm{m}$ ) the lumped $L M C$ and the distributed $L M C$ models are found to be in qualitative agreement but not quantitative; the Manakov approximations are not applicable in this regime. Importantly, effective SNR results in Fig. 3 show that performance degrades with $X T$ before it finally improves when approaching the SC-regime (a behavior also observed in [53]). As $X T$ increases from the WC-regime to the intermediate LMC regime, additional phase rotations introduced by LMC allow inter-modal four-wave-mixing phase matching to be achieved for a broader range of frequency combinations than it would be possible in the absence of LMC. Therefore, degrading performance since no significant averaging of the nonlinear coefficients is introduced. Moreover, partial phase matching over a broader range of frequency combinations means that the additional nonlinear penalty grows significantly with the number of WDM channels, as the results in Fig. 3 confirm. Finally, by increasing $X T$ towards the SC-regime, fast random rotations of the hyper-polarization state of the field along the fiber reduce the efficiency of the overall nonlinear process, averaging the nonlinear coefficients, improving performance. In summary, both the distributed LMC and lumped LMC models capture the performance dip with $X T$, however the lumped $L M C$ model due to its assumptions shows an artificial step degradation for values around $-55 \mathrm{~dB} / \mathrm{m}$. Therefore, in the following the distributed LMC model is taken as the reference.

To further evaluate the applicability of the different methods, Fig. 4 shows the effective $S N R$ error in $d B$ (given by $\triangle S N R_{x}=S N R_{x}-S N R_{\text {dist }-L M C}$, where $x$ can be Intra-DBP, WCDBP or SC-DBP) as a function of $X T$ for a wide range of $D M D$ values, 1 -to- $256 \mathrm{ps} / \mathrm{km}$, and a wide range of $X T$ values $-90-$ to- $0 \mathrm{~dB} / \mathrm{m}$. In general, it can be seen that the effective $S N R$ error increases with $D M D$, in particular for the Manakov approximation in the SC-regime. In Fig. 4-(a), for extremely

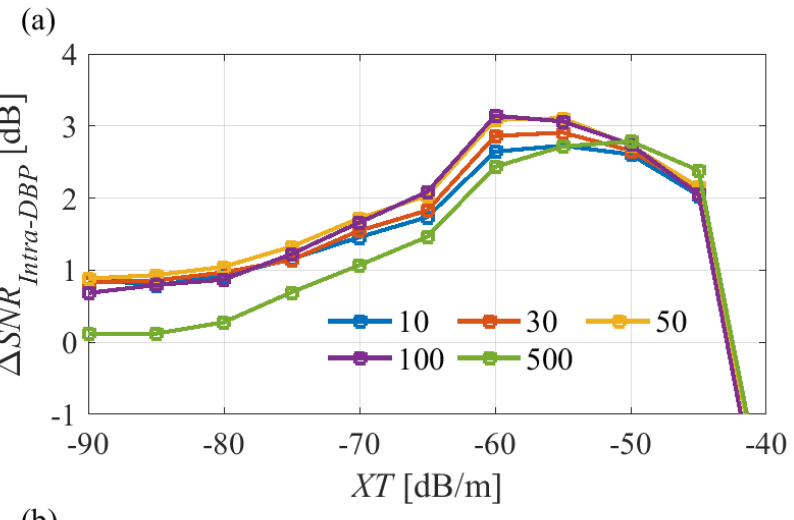

(b)

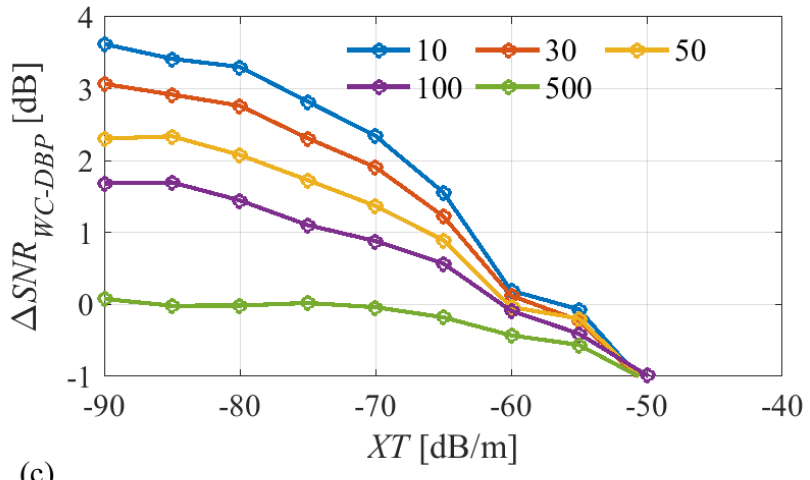

(c)

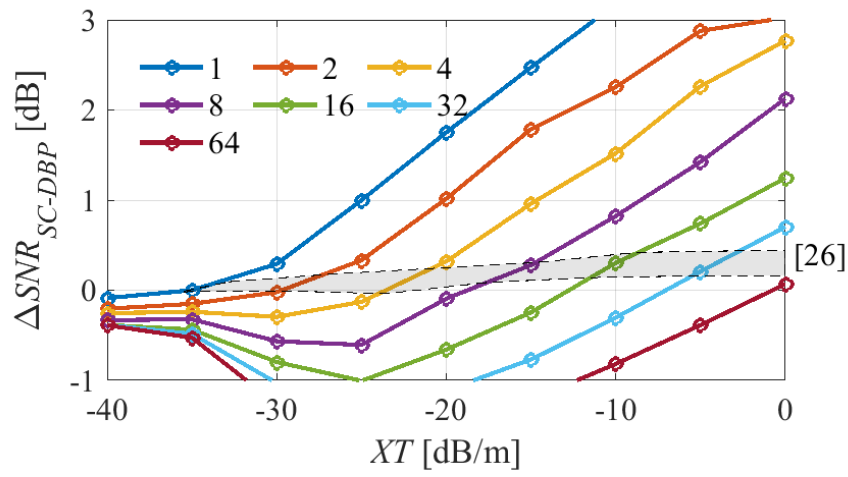

Fig. 5. Effective $S N R$ gain as a function of $X T$ after $7 \times 35 \mathrm{~km}, 0 \mathrm{dBm} / \mathrm{ch}$ and different $D M D$ values, with: (a) intra-DBP, (b) WC-DBP and (c) SC-DBP. Data points averaged over 10 repetitions (standard deviation was found to be around $0.2 \mathrm{~dB}$ for average values around $1 \mathrm{~dB}$ ).

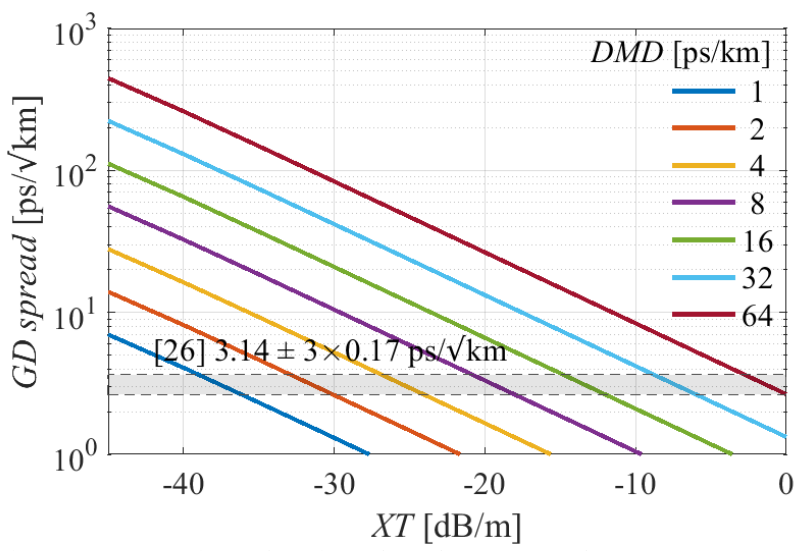

Fig. 6. GD spread as a function of $X T$ for a range of $D M D$ values matching Fig. 5-(c).

small $X T$ values $(<-70 \mathrm{~dB} / \mathrm{m})$, WC-Manakov generates accurate results even for $D M D$ as high as $256 \mathrm{ps} / \mathrm{km}$, thus 
mostly relevant for uncouple-core MCFs as practical FMFs have $X T \geq-70 \mathrm{~dB} / \mathrm{m}$. On the other side, for high $X T$ values (>-30 dB/m), Fig. 4-(a) shows that SC-Manakov is accurate for $D M D<10 \mathrm{ps} / \mathrm{km}$. However, for high $D M D$ values such as $256 \mathrm{ps} / \mathrm{km}$ SC-Manakov is only accurate for extremely high $X T$ values over $0 \mathrm{~dB} / \mathrm{m}$. More specifically, by looking at the $G D$ spread results overlapped in Fig. 4-(a), it can be concluded that SC-Manakov is accurate if the GD spread is smaller than $10 \mathrm{ps} / \sqrt{ } \mathrm{km}$. Therefore, SC-Manakov is mainly applicable to coupled-core MCFs [26]. On the other hand, Fig. 4-(b) shows that the lumped LMC model is able to accurately model propagation for $X T>-30 \mathrm{~dB} / \mathrm{m}$ even for $D M D$ over $100 \mathrm{ps} / \mathrm{km}$, thus widely applicable to the coupled-core MCFs presented in literature [26]. Finally, in the intermediate coupling regime $(-70 \mathrm{~dB} / \mathrm{m}$ to $-30 \mathrm{~dB} / \mathrm{m})$, Fig. $4-(\mathrm{a})$ and (b) show that only a distributed LMC model capable of introducing controllable LMC over small step-sizes (i.e. much smaller than the nonlinear effective length) can accurately model transmission.

\section{DBP PERFORMANCE}

This section analyses the performance of different DBP approaches for the system in section III (see Fig. 2) with 19 channels and 7 spans of $35 \mathrm{~km}$. Here, forward propagation is implemented following only the distributed LMC model. Instead, backward propagation is implemented following only averaged propagation (Manakov), using three different sets of nonlinear coefficients to best approximate the impact of LMC: (i) the WC-Manakov coefficients (WC-DBP); (ii) the SCManakov coefficients (SC-DBP); (iii) just the intra-modal nonlinear coefficients in WC-Manakov approximation (intraDBP). The coefficients are obtained by applying (3) and (4) to table II. To solve (2), the split-step Fourier method is implemented for: (i) forward transmission simulation with an adaptative step-size set by bounding the local error to be $<10^{-5}$, as in the previous section; (ii) DBP with a fix step-size of $100 \mathrm{~m}$ as smaller step-sizes led to negligible improvement. Regarding the GD operator in DBP: WC-DBP and intra-DBP consider the uncoupled GD vector (constant for the whole transmission), and SC-DBP neglects differential group delay.

DBP performance is known to be strongly depend on the number of channels back-propagated [61] and on the effective number of bits $(\mathrm{ENoB})$ of the analog-to-digital convertors (ADC) in the receiver front-end [62]. Therefore, in this section we consider: (i) state-of-the-art analog-to-digital converters with an effective number of bits equal to 5.5 bits, (ii) backpropagation of a sub-set of channels spanning from single channel to all channels transmitted. Note that DBP computational complexity scales linearly with the number of back-propagated channels [63].

Figure 5 shows the effective SNR improvement over linear equalization as a function of $X T$ after $245 \mathrm{~km}$ with different values of $D M D$ and a launch power of $0 \mathrm{dBm} / \mathrm{ch}$, for: (a) intra-, (b) WC- and (c) SC-DBP. In this case all 19 channels were back-propagated in order to establish a DBP performance baseline without a compromise on the number of channels. In Fig. 5-(a) and -(b) simulations considered GD-managed spans by cascading 2 fibers with opposite sign GD vectors, while in Fig. 5-(c) non-GD-managed spans are considered. In all cases,
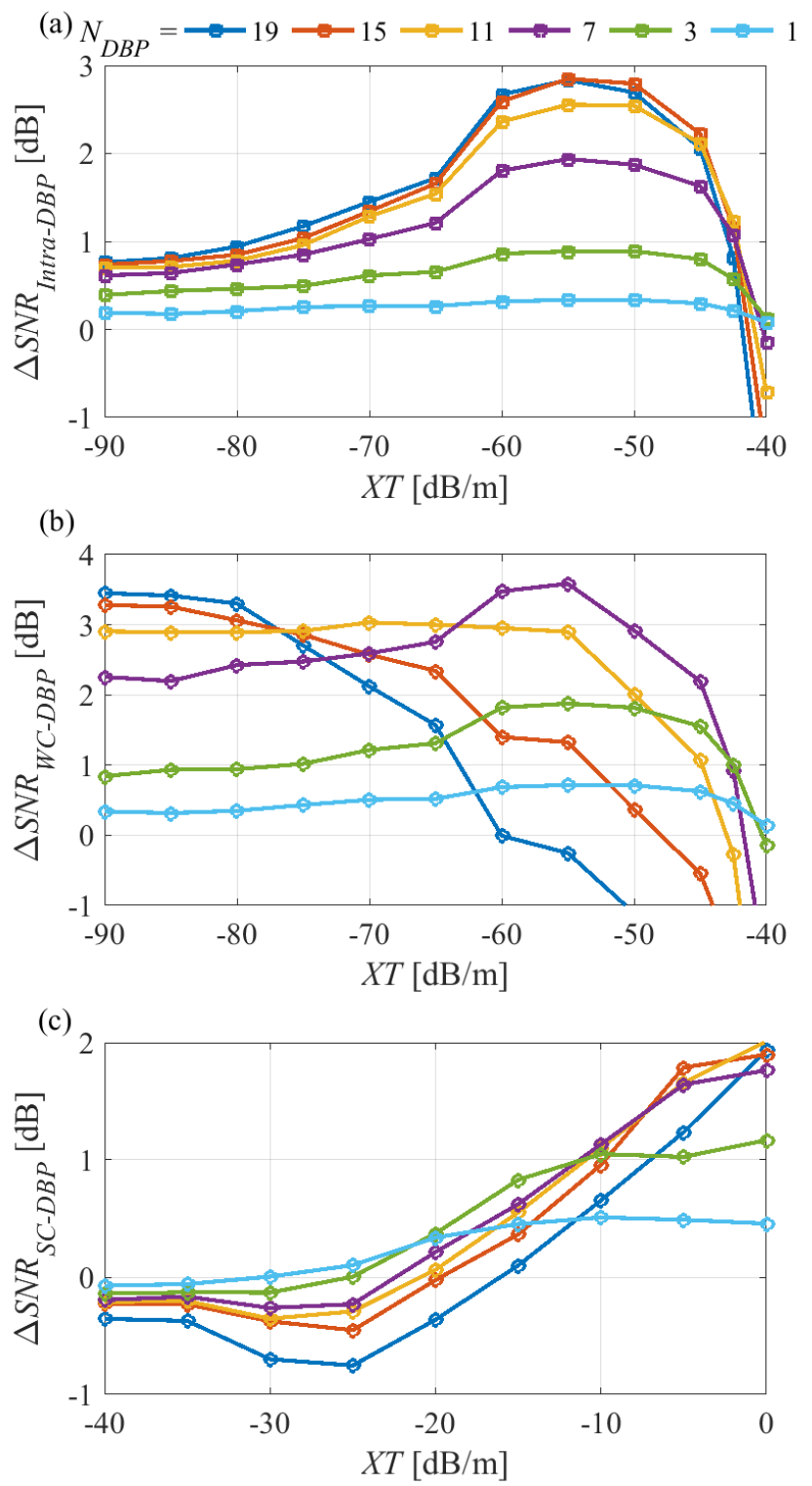

Fig. 7. Effective $S N R$ gain as a function of $X T$ after $7 \times 35 \mathrm{~km}, 0 \mathrm{dBm} / \mathrm{ch}$, $D M D=0 \mathrm{ps} / \mathrm{km}$, for different numbers of back-propagated channels, with: (a) intra-DBP, (b) WC-DBP and (c) SC-DBP. Data points averaged over 10 repetitions (standard deviation was found to be around $0.2 \mathrm{~dB}$ for average values around $1 \mathrm{~dB}$ ).

a broad range of $D M D$ and $X T$ values are considered to cover a wide variety of operational regimes. The launching power is selected to be $0 \mathrm{dBm} / \mathrm{ch}(2 \mathrm{~dB}$ above the optimum launch power with DBP according to further simulation results) such that performance is dominated by fiber nonlinearities, in this way maximizing the DBP performance. Note that at the respective optimum launch powers the DBP gain in the following would be smaller, comparable to the DBP gain in single-mode fibers [64].

Fig. 5-(a) to -(c) show that all DBP techniques implemented can provide significant nonlinear compensation (above $1 \mathrm{~dB}$ ) over specific operational regimes. WC- and SC-DBP provide significant compensation in the regimes where Manakov approximations are valid, this is for $X T<-70 \mathrm{~dB} / \mathrm{m}$ and $X T>-35 \mathrm{~dB} / \mathrm{m}$. Interestingly, Fig. 5-(a) and -(b) show that intra-DBP provides a performance improvement in many cases higher than that of WC-DBP. Intra-DBP performs particularly 
well when inter-modal nonlinear processes are not dominant, this is the case for non-negligible $D M D$ and intermediate $X T$ such that inter-modal four-wave-mixing phase matching is poor. Conversely, intra-DBP under-performs WC-DBP when inter-modal nonlinear processes are significant, this is for sufficiently low $X T$ intra-DBP gain rolls-off as can be seen in Fig. 5-(a). In this way, for the intermediate LCM regime, with $-60<X T[\mathrm{~dB} / \mathrm{m}]<-45$ and $D M D \geq 10 \mathrm{ps} / \mathrm{km}$ intra-DBP provides the highest improvement between 1 and $3 \mathrm{~dB}$, and for fibers with $X T<-60 \mathrm{~dB} / \mathrm{m}$ and $D M D \leq 100 \mathrm{ps} / \mathrm{km}$ WC-DBP provides an improvement between 1 and $3.5 \mathrm{~dB}$. These $X T$ and $D M D$ ranges cover many the fibers presented in literature [2025].

In the SC-regime, Fig. 5-(c) shows that SC-DBP can provide significant nonlinear compensation for a significant range of (uncoupled) $D M D$ and $X T$ values. This range is better captured by looking at $G D$ spread (see section II.B). It is shown in Fig. 6 as function of $X T$ for the same range of $D M D$ values as in Fig. 5-(c). In Fig. 5-(c) and Fig. 6, the grey shaded area delimited by a dashed black line, bounds the possible working area (3 standard deviations) for the CC- MCF presented in [26] with a $G D$ spread of $3.14 \pm 0.17 \mathrm{ps} / \sqrt{ } \mathrm{km}$ (average and standard deviation). Within such scenario performance improvement can reach $0.5 \mathrm{~dB}$. Finally, Fig. 5-(c) shows that further reduction of the $G D$ spread can unlock a potential for $2 \mathrm{~dB}$ improvement.

In the intermediate $\mathrm{LMC}$ regime, there is a range of $X T$ values $(-45 \mathrm{~dB} / \mathrm{m}$ to $-35 \mathrm{~dB} / \mathrm{m})$ over which none of the DBP approaches studied work even for negligible $D M D$. This is because for significant transmission distances $(245 \mathrm{~km}$, in this case) LMC leads to evolutions of the nonlinear operator that differ significantly from that of the uncoupled operator and from the Manakov approximation. Away from the operational regime identified for WC- and SC-DBP, the evolution of the GD operator is no longer well approximated using the uncoupled GD coefficients, thus the nonlinear distortion is either overcompensated or undercompensated when using the uncoupled nonlinear coefficients.

Fig. 7 shows the effective SNR gain offered by DBP when back-propagating a sub-set of channels $([19,11,7,3,1]$ channels), $D M D=10 \mathrm{ps} / \mathrm{km}$, non-GD-managed spans, for: (a) Intra-DBP, (b) WC-DBP and (c) SC-DBP. In this case the trade-off between DBP computational complexity and effective SNR gain is directly targeted. Fig. 7 (a) shows that Intra-DBP performance improves with the number of back-propagated channels, it is maximum considering all 19 channels transmitted, as expected. However, in Fig. 7 (b) this is not the case. The reason is that for a significant among of $D M D$ $(10 \mathrm{ps} / \mathrm{km})$, in the intermediate LMC regimes, the coupling experienced by each channel is decorrelated [7]. In this case, back-propagating while considering most of the centre channel neighbours added uncorrelated nonlinear interference instead of anti-correlated nonlinear interference - increasing the total nonlinear penalty. At $X T=-55 \mathrm{~dB} / \mathrm{m}$, well within the intermediate LMC regime, the optimum number of backpropagated channels is 7 channels, while 19 channels leads to the worst performance. Fig. 7 (c) leads to similar conclusions in the SC-regime with SC-DBP. To the best of our knowledge this is the first time that back-propagating of a sub-group of channels in WDM-SDM systems has been shown to lead to better performance than back-propagating all channels.

\section{CONCLUSION}

This work shows that even for the complex spatial multiplexed systems under challenging LMC and DMD conditions there is significant potential for performance improvement using DBP. To maximize DBP performance appropriate approximations for the effect of the stochastic nature of the LMC have to be considered. For example, fibers optimized primarily for low $X T$ (and with intermediate-to-high $D M D$ ), including trenchassisted graded-index fibers [21] or multiple-step index fibers [24], allow a significant DBP gain if inter-modal nonlinear processes are neglected. However, this signal processing approach gives no gain for high $X T$ (and low $D M D$ ) fibers such as CC-MCFs [26]. Instead, the so called generalized Manakov approach provides significant gains. Moreover, it was shown that for systems with significant $D M D$ operating in the intermediate LMC regime, DBP performance can be improved by reducing the number of back propagated channels. Finally, whilst a small range of possible fiber parameters exist where the approximate models considered here failed to provide significant gain, and compensation would require continuous estimation of the random LMC, significant performance gains were possible for all possible regimes in which real fibers operate.

\section{REFERENCES}

[1] D. J. Richardson, J. M. Fini, and L. E. Nelson, "Space-division multiplexing in optical fibres," Nature Photonics, Review Article vol. 7, p. 354, 04/29/online 2013.

[2] L. Bigot, G. L. Cocq, and Y. Quiquempois, "Few-Mode ErbiumDoped Fiber Amplifiers: A Review," Journal of Lightwave Technology, vol. 33, no. 3, pp. 588-596, 2015.

[3] P. J. Winzer and D. T. Neilson, "From Scaling Disparities to Integrated Parallelism: A Decathlon for a Decade," Journal of Lightwave Technology, vol. 35, no. 5, pp. 1099-1115, 2017.

[4] D. M. Marom et al., "Survey of photonic switching architectures and technologies in support of spatially and spectrally flexible optical networking [invited]," IEEE/OSA Journal of Optical Communications and Networking, vol. 9, no. 1, pp. 1-26, 2017.

[5] F. Ferreira, P. Monteiro, and H. Silva, "Semi-analytical model for linear modal coupling in few-mode fiber transmission," in 2012 14th International Conference on Transparent Optical Networks (ICTON), 2012: IEEE.

[6] K.-P. Ho and J. M. Kahn, "Linear Propagation Effects in ModeDivision Multiplexing Systems," Journal of Lightwave Technology, vol. 32, no. 4, pp. 614-628, 2014.

[7] A. Mecozzi, C. Antonelli, and M. Shtaif, "Intensity impulse response of SDM links," Opt Express, vol. 23, no. 5, pp. 5738-43, Mar 92015.

[8] C. Antonelli, A. Mecozzi, and M. Shtaif, "The delay spread in fibers for SDM transmission: dependence on fiber parameters and perturbations," Opt Express, vol. 23, no. 3, pp. 2196-202, Feb 9 2015.

[9] F. M. Ferreira, C. S. Costa, S. Sygletos, and A. D. Ellis, "SemiAnalytical Modelling of Linear Mode Coupling in Few-Mode Fibers," Journal of Lightwave Technology, vol. 35, no. 18, pp. 40114022, 2017.

[10] J. Vuong, P. Ramantanis, A. Seck, D. Bendimerad, and Y. Frignac, "Understanding discrete linear mode coupling in few-mode fiber transmission systems," in 2011 37th European Conference and Exhibition on Optical Communication, 2011, pp. 1-3.

[11] A. Mecozzi, C. Antonelli, and M. Shtaif, "Coupled Manakov equations in multimode fibers with strongly coupled groups of modes," Optics Express, vol. 20, no. 21, pp. 23436-23441, 2012/10/08 2012 . 
[12] A. Mecozzi, C. Antonelli, and M. Shtaif, "Nonlinear propagation in multi-mode fibers in the strong coupling regime," Optics Express, vol. 20, no. 11, pp. 11673-11678, 2012/05/21 2012.

[13] S. Mumtaz, R.-J. Essiambre, and G. P. Agrawal, "Nonlinear Propagation in Multimode and Multicore Fibers: Generalization of the Manakov Equations," Journal of Lightwave Technology, vol. 31, no. 3, pp. 398-406, 2013

[14] F. Ferreira, S. Jansen, P. Monteiro, and H. Silva, "Nonlinear semianalytical model for simulation of few-mode fiber transmission," IEEE Photonics Technology Letters, vol. 24, no. 4, pp. 240--242, 2 2012.

[15] C. Koebele, M. Salsi, G. Charlet, and S. Bigo, "Nonlinear Effects in Mode-Division-Multiplexed Transmission Over Few-Mode Optical Fiber," IEEE Photonics Technology Letters, vol. 23, no. 18, pp. 1316-1318, 2011.

[16] F. Poletti and P. Horak, "Description of ultrashort pulse propagation in multimode optical fibers," Journal of the Optical Society of America B, vol. 25, no. 10, pp. 1645-1654, 2008/10/01 2008.

[17] A. Lobato et al., "Impact of mode coupling on the mode-dependent loss tolerance in few-mode fiber transmission," Opt. Express, vol. 20, no. 28, p. 29776, 20122012.

[18] K.-P. Ho and J. M. Kahn, "Mode-dependent loss and gain: statistics and effect on mode-division multiplexing," Optics Express, vol. 19, no. 17, pp. 16612-16635, 2011/08/15 2011.

[19] C. Antonelli, A. Mecozzi, M. Shtaif, and P. J. Winzer, "Modeling and performance metrics of MIMO-SDM systems with different amplification schemes in the presence of mode-dependent loss," Optics Express, vol. 23, no. 3, pp. 2203-2219, 2015/02/09 2015.

[20] L. An, A. A. Amin, C. Xi, and W. Shieh, "Reception of mode and polarization multiplexed 107-Gb/s CO-OFDM signal over a twomode fiber," in 2011 Optical Fiber Communication Conference and Exposition and the National Fiber Optic Engineers Conference, 2011, pp. 1-3.

[21] L. Gruner-Nielsen et al., "Few Mode Transmission Fiber With Low DGD, Low Mode Coupling, and Low Loss," Journal of Lightwave Technology, vol. 30, no. 23, pp. 3693-3698, 2012.

[22] R. Ryf et al., "Mode-Division Multiplexing Over $96 \mathrm{~km}$ of FewMode Fiber Using Coherent 6\$, Itimesl,\$6 MIMO Processing," Journal of Lightwave Technology, vol. 30, no. 4, pp. 521-531, 2012.

[23] R. Ryf et al., "Space-Division Multiplexed Transmission over 4200 km 3-Core Microstructured Fiber," in National Fiber Optic Engineers Conference, Los Angeles, California, 2012, p. PDP5C.2: Optical Society of America.

[24] T. Sakamoto, T. Mori, T. Yamamoto, and S. Tomita, "Differential Mode Delay Managed Transmission Line for WDM-MIMO System Using Multi-Step Index Fiber," Journal of Lightwave Technology, vol. 30, no. 17, pp. 2783-2787, 2012

[25] T. Mori, T. Sakamoto, M. Wada, T. Yamamoto, and F. Yamamoto, "Low DMD four LP mode transmission fiber for wide-band WDMMIMO system," in 2013 Optical Fiber Communication Conference and Exposition and the National Fiber Optic Engineers Conference (OFC/NFOEC), 2013, pp. 1-3.

[26] T. Hayashi, Y. Tamura, T. Hasegawa, and T. Taru, "Record-Low Spatial Mode Dispersion and Ultra-Low Loss Coupled Multi-Core Fiber for Ultra-Long-Haul Transmission," Journal of Lightwave Technology, vol. 35, no. 3, pp. 450-457, 2017.

[27] C. S. Costa, F. M. Ferreira, N. M. Suibhne, S. Sygletos, and A. D. Ellis, "Receiver Memory Requirement in Mode Delay Compensated Few-Mode Fibre Spans with Intermediate Coupling," in ECOC 2016; 42nd European Conference on Optical Communication, 2016, pp. 1-3.

[28] S. Ö. Arik, D. Askarov, and J. M. Kahn, "Effect of Mode Coupling on Signal Processing Complexity in Mode-Division Multiplexing," Journal of Lightwave Technology, vol. 31, no. 3, pp. 423-431, 2013.

[29] R. Ryf et al., "Long-Distance Transmission over Coupled-Core Multicore Fiber," in ECOC 2016 - Post Deadline Paper; 42nd European Conference on Optical Communication, 2016, pp. 1-3.

[30] F. Ferreira, C. Sanchez, N. Suibhne, S. Sygletos, and A. Ellis, "Nonlinear Transmission Performance in Delay-Managed FewMode Fiber Links with Intermediate Coupling," in Optical Fiber Communication Conference, Los Angeles, California, 2017, p. Th2A.53: Optical Society of America.

[31] G. Rademacher et al., "Long-Haul Transmission over Few-Mode Fibers with Space-Division Multiplexing," Journal of Lightwave Technology, vol. PP, no. 99, pp. 1-1, 2017.
A. D. Ellis, N. Mac Suibhne, F. C. Gunning, and S. Sygletos, "Expressions for the nonlinear transmission performance of multimode optical fiber," Opt Express, vol. 21, no. 19, pp. 22834-46, Sep 232013.

[33] N. M. Suibhne, A. D. Ellis, F. C. G. Gunning, and S. Sygletos, "Experimental verification of four wave mixing efficiency characteristics in a few mode fibre," in 39th European Conference and Exhibition on Optical Communication (ECOC 2013), 2013, pp. $1-3$.

[34] Z. S. Eznaveh, J. E. Antonio-Lopez, J. C. A. Zacarias, A. Schülzgen, C. M. Okonkwo, and R. A. Correa, "All-fiber few-mode multicore photonic lantern mode multiplexer," Optics Express, vol. 25, no. 14, pp. 16701-16707, 2017/07/10 2017.

[35] K. Shikama, Y. Abe, H. Ono, and A. Aratake, "Low-Loss and LowMode-Dependent-Loss Fan-In/Fan-Out Device for 6-Mode 19-Core Fiber," Journal of Lightwave Technology, vol. 36, no. 2, pp. 302308, 2018.

[36] S. Bade et al., "Fabrication and Characterization of a Modeselective 45-Mode Spatial Multiplexer based on Multi-Plane Light Conversion," in Optical Fiber Communication Conference Postdeadline Papers, San Diego, California, 2018, p. Th4B.3: Optical Society of America.

[37] N. Riesen, S. Gross, J. D. Love, Y. Sasaki, and M. J. Withford, "Monolithic mode-selective few-mode multicore fiber multiplexers," Scientific Reports, vol. 7, no. 1, p. 6971, 2017/08/01 2017.

[38] S. Kruk et al., "Transparent Dielectric Metasurfaces for Spatial Mode Multiplexing," Laser \& Photonics Reviews, vol. 12, no. 8, p. 1800031, 2018.

[39] F. Ferreira, N. MacSuibhne, C. Sánchez, S. Sygletos, and A. D. Ellis, "Advantages of strong mode coupling for suppression of nonlinear distortion in few-mode fibers," in Optical Fiber Communication Conference 2016, 2016: Optical Society of America.

[40] F. M. Ferreira, N. MacSuibhne, S. Sygletos, and A. D. Ellis, "Fewmode fibre group-delays with intermediate coupling," in European Conference on Optical Communication, ECOC, United States, 2015: IEEE.

[41] F. Ferreira, S. Sygletos, A. Ellis, and N. J. Doran, "Digital Back Propagation Performance in Spatial Multiplexing Systems," in European Conference on Optical Communication, Dublin, Ireland, 2019, p. W.3.A.2.

[42] F. M. Ferreira, C. S. Costa, S. Sygletos, and A. D. Ellis, "Overcoming degradation in spatial multiplexing systems with stochastic nonlinear impairments," Scientific Reports, vol. 8, no. 1, p. $17539,2018 / 12 / 032018$.

[43] F. M. Ferreira, C. S. Costa, S. Sygletos, and A. D. Ellis, "Nonlinear Performance of Few-Mode Fiber Links With Intermediate Coupling," Journal of Lightwave Technology, vol. 37, no. 3, pp. 989-999, 2019/02/01 2019.

[44] Q. Sui et al., "Long-haul quasi-single-mode transmissions using few-mode fiber in presence of multi-path interference," Optics Express, vol. 23, no. 3, pp. 3156-3169, 2015/02/09 2015.

[45] T. Hayashi, T. Taru, O. Shimakawa, T. Sasaki, and E. Sasaoka, "Uncoupled multi-core fiber enhancing signal-to-noise ratio," Optics Express, vol. 20, no. 26, pp. B94-B103, 2012/12/10 2012.

[46] I. Kaminow, "Polarization in optical fibers," IEEE Journal of Quantum Electronics, vol. 17, no. 1, pp. 15-22, 1981.

[47] G. J. Foschini and C. D. Poole, "Statistical theory of polarization dispersion in single mode fibers," Journal of Lightwave Technology, vol. 9, no. 11, pp. 1439-1456, 1991.

[48] P. K. A. Wai and C. R. Menyuk, "Polarization mode dispersion, decorrelation, and diffusion in optical fibers with randomly varying birefringence," Journal of Lightwave Technology, vol. 14, no. 2, pp. 148-157, 1996.

[49] C. Antonelli, A. Mecozzi, M. Shtaif, and P. J. Winzer, "Stokes-space analysis of modal dispersion in fibers with multiple mode transmission," Optics Express, vol. 20, no. 11, pp. 11718-11733, 2012/05/21 2012.

[50] F. Ferreira, S. Sygletos, and A. Ellis, "Impact of linear mode coupling on the group delay spread in few-mode fibers," in 2015 Optical Fiber Communications Conference and Exhibition (OFC), 2015, pp. 1-3.

[51] O. V. Sinkin, R. Holzlohner, J. Zweck, and C. R. Menyuk, "Optimization of the split-step fourier method in modeling optical- 
fiber communications systems," Journal of Lightwave Technology, vol. 21, no. 1, pp. 61-68, 2003.

[52] D. Marcuse, C. R. Manyuk, and P. K. A. Wai, "Application of the Manakov-PMD equation to studies of signal propagation in optical fibers with randomly varying birefringence," Journal of Lightwave Technology, vol. 15, no. 9, pp. 1735-1746, 1997.

[53] S. Buch, S. Mumtaz, R.-J. Essiambre, A. M. Tulino, and G. P. Agrawal, "Averaged nonlinear equations for multimode fibers valid in all regimes of random linear coupling," Optical Fiber Technology, vol. 48, pp. 123-127, 2019/03/01/ 2019.

[54] C. Antonelli, M. Shtaif, and A. Mecozzi, "Modeling of Nonlinear Propagation in Space-Division Multiplexed Fiber-Optic Transmission," Journal of Lightwave Technology, vol. 34, no. 1, pp. 36-54, 2016.

[55] F. Ferreira, D. Fonseca, and H. J. A. Silva, "Design of few-mode fibers with M-modes and low differential mode delay," Journal of Lightwave Technology, vol. 32, no. 3, pp. 353--360, 22014.

[56] O.150 - Series O: Specifications of measuring equipment, 1996.

[57] E. Mateo, L. Zhu, and G. Li, "Impact of XPM and FWM on the digital implementation of impairment compensation for WDM transmission using backward propagation," Optics Express, vol. 16, no. 20, pp. 16124-16137, 2008/09/29 2008.

[58] E. Ip and J. M. Kahn, "Compensation of Dispersion and Nonlinear Impairments Using Digital Backpropagation," Journal of Lightwave Technology, vol. 26, no. 20, pp. 3416-3425, 2008.

[59] A. Alvarado, T. Fehenberger, B. Chen, and F. M. J. Willems, "Achievable Information Rates for Fiber Optics: Applications and Computations," Journal of Lightwave Technology, vol. 36, no. 2, pp. 424-439, 2018.

[60] N. J. Doran and A. D. Ellis, "Minimising total energy requirements in amplified links by optimising amplifier spacing," Optics Express, vol. 22, no. 16, pp. 19810-19817, 2014/08/11 2014.

[61] G. Liga, T. Xu, A. Alvarado, R. I. Killey, and P. Bayvel, "On the performance of multichannel digital backpropagation in highcapacity long-haul optical transmission," Optics Express, vol. 22, no. 24, pp. 30053-30062, 2014/12/01 2014.

[62] L. Galdino et al., "On the limits of digital back-propagation in the presence of transceiver noise," Optics Express, vol. 25, no. 4, pp. 4564-4578, 2017/02/20 2017.

[63] O. Sidelnikov, A. Redyuk, and S. Sygletos, "Equalization performance and complexity analysis of dynamic deep neural networks in long haul transmission systems," Optics Express, vol. 26, no. 25, pp. 32765-32776, 2018/12/10 2018.

[64] C. B. Czegledi et al., "Digital backpropagation accounting for polarization-mode dispersion," Optics Express, vol. 25, no. 3, p. 1903, 2017. 